Fibre Bragg grating sensors for monitoring the metal inert gas and friction stir welding processes

This article has been downloaded from IOPscience. Please scroll down to see the full text article.

2010 Meas. Sci. Technol. 21085105

(http://iopscience.iop.org/0957-0233/21/8/085105)

View the table of contents for this issue, or go to the journal homepage for more

Download details:

IP Address: 193.136.33.229

The article was downloaded on 29/06/2010 at 17:54

Please note that terms and conditions apply. 


\title{
Fibre Bragg grating sensors for monitoring the metal inert gas and friction stir welding processes
}

\author{
V Richter-Trummer ${ }^{1}$, S O Silva ${ }^{2}$, D F C Peixoto ${ }^{1}$, O Frazão ${ }^{2}$, \\ P M G P Moreira ${ }^{3}$, J L Santos ${ }^{2}$ and P M S T de Castro ${ }^{1}$ \\ ${ }^{1}$ FEUP/IDMEC, Rua Dr Roberto Frias, 4200-465 Porto, Portugal \\ 2 INESC Porto, Campus da FEUP, Rua Dr Roberto Frias 378, 4200-465 Porto, Portugal \\ ${ }^{3}$ INEGI, Campus da FEUP, Rua Dr Roberto Frias, 4200-465 Porto, Portugal \\ E-mail: valentin@fe.up.pt
}

Received 7 April 2010, in final form 27 May 2010

Published 29 June 2010

Online at stacks.iop.org/MST/21/085105

\begin{abstract}
Fibre Bragg grating (FBG) sensors are finding increased usage in experimental mechanics for monitoring service conditions in structures and other equipment and are currently being tested for process monitoring. In FBG sensors, strain and temperature cause a shift in the Bragg wavelength reflected by the grating contained in these fibres. In situ monitoring of strain and temperature during welding processes increases knowledge of the welded material and the welding process itself. In the present work, two welding processes are monitored using FBG sensors and the complete measurement approach including sensor selection, calibration, instrumentation, welding monitoring and result interpretation is presented. Calibration for strain measurements at constant temperature was performed using a four-point bending test, and temperature calibration was carried out using an oven. Results for a sensor length of $5 \mathrm{~mm}$ are presented. Both transient and residual strains were recorded during experiments on metal inert gas and friction stir welding and the possible impact of this monitoring technology is discussed in the light of process optimization and subsequent structural health monitoring.
\end{abstract}

Keywords: FBG sensor, FSW, MIG, strain and temperature measurement, welding monitoring

(Some figures in this article are in colour only in the electronic version)

\section{Introduction}

Some of the unique advantages of fibre Bragg grating (FBG)-based fibre optic sensors are the immunity to electromagnetic and radio frequency interference (EMI and $\mathrm{RFI}$ ), the light weight, the capability of intermittent readings with reconnection between readings and the wavelength multiplexing capability, as described in the ISIS design manual for FBG sensors [1].

Applications are frequently found in civil engineering, such as monitoring of bridges. While Barbosa et al [2] demonstrated the use of FBG sensors for monitoring a newly built bridge, Figueiras et al [3] applied this monitoring technique to a 19th century metallic bridge. Due to frequency coding of the measurements, the output does not depend on the signal intensity. This allows very long distances for data transmission, while still retaining adequate signal strength [1]. This capability may be favourable for the final application of FBG-based sensors for structural health monitoring (SHM) in large aeronautical and railroad cars. As stated by Thursby et al [4], the knowledge of the load history of a part of a structure can provide valuable data for the prediction of its lifetime.

If the load history includes not only the loads applied during the service-life but also loads applied during manufacturing, a more complete picture can be drawn about the real state of a structure, including residual stresses which may be present in the structure at the beginning of the in-service-life. For this information to be included, instrumentation has to be applied before the part is produced, 
as in the welding processes dealt with in the present paper. The present paper is a first step in that direction.

Data on the application of FBG sensors for monitoring welding are very scarce and concern fusion processes only. Suárez et al [5] presented a first attempt to monitor weldinginduced strains using FBG sensors, where temperature measurements were validated using thermocouples but the strain results were not compared with measurements made using alternative techniques. Suárez et al [5] present results for the plate side opposite to the weld bead only, and the grating length is not given in that paper. Later on, Moreira et al [6] presented further results concerning temperature monitoring using $30 \mathrm{~mm}$ long gratings, validated using thermocouples and also thermography, on the weld bead side of the plate.

The present work seeks to further explore the application of FBG sensors in welding monitoring, optimizing the sensor application techniques and calibration procedures, and systematically comparing FBG measurements using $5 \mathrm{~mm}$ long gratings with data obtained using alternative techniques. The present results concern the weld bead side of the plate, in the case of metal inert gas (MIG), and the shoulder side in the case of friction stir welding (FSW). The final intention is to enhance the knowledge concerning this application of FBG sensors that may become increasingly interesting as fibre optic technology progressively becomes more widely used in industry.

\subsection{FBG sensors}

Ultraviolet (UV) light at $248 \mathrm{~nm}$ passing through a phase mask for about 30-40 s writes a sensor at a specified wavelength. Using the pulsed output of a laser together with an appropriate phase mask, the UV light changes the refractive index of the Ge-doped core at precisely spaced periodical locations creating a well-defined grating [1]. During the welding experiments the fibres are protected from UV light. This way the grating only reflects light at the inscribed central Bragg wavelength $\lambda_{B}$.

Due to physical changes of the sensing device, such as the application of strain or temperature, the pitch of the grating is changed, and therefore the measured wavelength is also shifted, reflecting the change in strain as can be seen in equation (1):

$$
\Delta \lambda_{B}=\lambda_{B}(1-P e) \Delta \varepsilon+\lambda_{B}(\alpha-\xi) \Delta T .
$$

$P e$ is the photoelastic coefficient, $\Delta \varepsilon$ is the strain variation, $\alpha$ is the thermal expansion coefficient, $\xi$ is the thermo-optic coefficient and $\Delta T$ is the temperature variation of the sensor.

One of the limitations of the Bragg grating technology is its cross sensitivity to temperature. Approaches to solve this problem under different conditions are mentioned by Ferraro and de Natale [7], and a solution based on a second grating free from mechanical strain was deemed appropriate for the current application. Therefore two Bragg structures were used at the same location, where the reference grating is sensitive to temperature only (unstrained) and the second one is sensitive to both strain and temperature.

In this case, it is possible to compensate for the temperature component of the second FBG sensing device.

\subsection{Sensor length and application technique}

FBG sensors may be produced with several grating lengths, typically between 5 and $200 \mathrm{~mm}$ [1], the longer gratings being particularly interesting for distributed sensing applications. In the present case the grating length was chosen to be short, since measurements were taken at points and not along lines. Furthermore, the available laser equipment and phase mask limit the possible grating lengths to approximately 5-30 mm. $10 \mathrm{~mm}$ and longer gratings have been used previously by the authors. When the strain gradient in a grating is too high, the wavelength peak will not only shift, but it will be divided, thus reinforcing the need for shorter grating lengths. Therefore $5 \mathrm{~mm}$ long gratings were produced and used in the present work.

The capability of distributed sensing using longer gratings should be kept in mind for future applications with high strain and temperature gradients. In the present case only the average strain between the first and last etched regions of the grating was measured and therefore no distributed sensing was performed.

The goal of this work was to measure the surface strain and temperature during the welding processes. No attempt was made to embed the fibres inside the plates.

For surface application, it is necessary to guarantee a good transmission of the physical quantities to be measured to the sensor itself. This is an important aspect which has been modeled by Duck et al [8], who also mentioned that less steep gradients lead to better strain transfer which reinforces the idea of using shorter grating lengths for a lower influence of the steep gradient on the measurements. For strain sensors this means that the sensor has to be adequately bonded along the complete grating length using a sufficiently flexible adhesive which is stiff enough to transmit all surface strains to the fibre. While one approach would be the use of a ceramic adhesive as used by Suárez et al [5], a better strain transfer from the plate surface to the sensing device can be obtained by the use of an epoxy-based high-temperature strain gauge adhesive. The inconvenience of this type of adhesive is however that temperatures should not be much higher than $200{ }^{\circ} \mathrm{C}$. In the present case the high-temperature strain gauge adhesive $\mathrm{M}$ Bond 600 from Vishay was selected for this task, since the cyanoacrylate-based alternatives which can be used at room temperature are not recommended for usage over $80^{\circ} \mathrm{C}$.

The temperature sensors, on the other hand, have to be insulated from strain effects, while retaining sufficient contact to the surface for a good heat transfer to the fibre. For this purpose, the fibre was only bonded to the surface with two dots. Due to its small curvature, no strain is transferred, and silver-based thermal paste was applied around the grating for enhanced heat transfer. The advantage of using a curved fibre instead of a straight fibre inside a very thin tube as used by Suárez et al [5] is the fact that strains are not transmitted to the grating, even if more than one sensor is located on the same fibre. When a straight sensor inside a capillary tube is used for sensing temperature, this sensor should be on the free end of the fibre for better isolation from mechanical strains. 

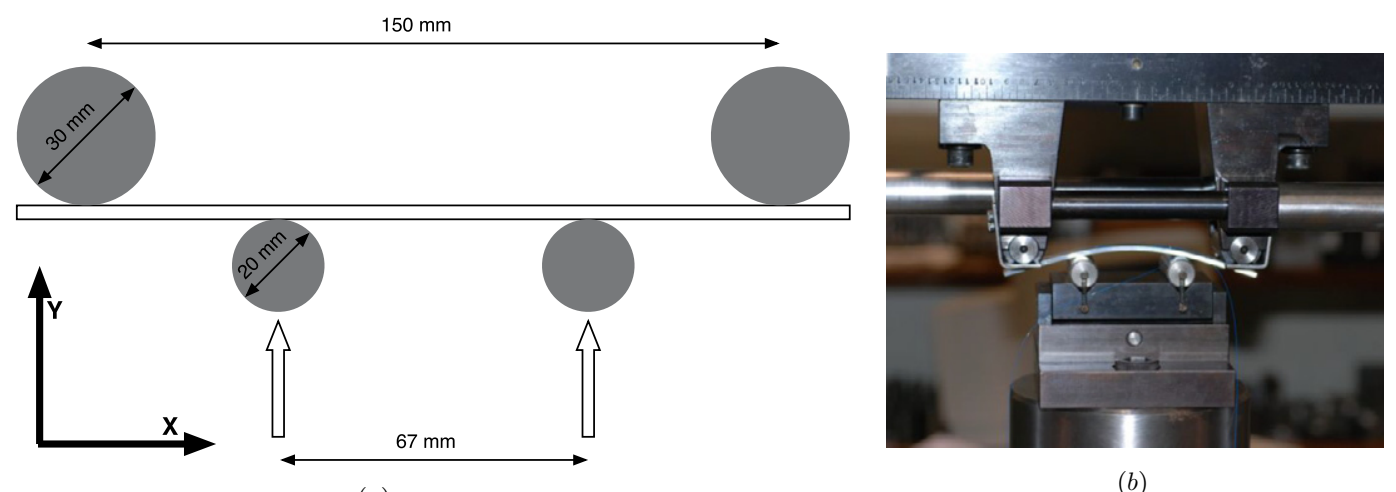

(a)

(b)

Figure 1. Experimental setup for the four-point bending test. (a) Schematic representation of the four-point bending test. (b) Photograph of the experiment.

\subsection{Data acquisition}

FBG interrogation was performed with a FiberSensing BraggMeter FS4200 interrogation unit, which is based on a tunable optical fibre laser that relies on a Fabry-Perot filter. In order to capture the temperature and strain peaks during the welding processes adequately, an acquisition rate of $200 \mathrm{~Hz}$ was used. Since the used interrogation concept is intrinsically amenable to FBG multiplexing, several FBG sensors were inscribed into a single fibre.

\subsection{Base material characterization}

The material used was the aluminium alloy AA6082-T6, with Young's modulus $(E)$ of $65 \mathrm{GPa}$ and a yield strength of $252 \mathrm{MPa}$ [9].

\section{Fibre Bragg grating calibration}

Only general calibration constants were available from the manufacturer of the gratings, which do not account for the proposed application technique. Before measurements can be performed in a welding process, the measuring devices have to be calibrated, so that the obtained results may be considered valid.

\subsection{Calibration for strain measurement}

The calibration for strain measurement at constant temperature was performed using the four-point bending specimens instrumented with FBG sensors and strain gauges, following the procedure discussed by Richter-Trummer et al [10], as shown in figure 1 .

This type of mechanical test provides adequate space for installation of instrumentation, and minimizes the strain gradient in the region measured. The bending moment is constant between inner rollers, and in the linear elastic regime, strain in the upper and lower surfaces is proportional to this moment [11]. If continued above the elastic limit, this type of test allows the measurement of a large range of tensile and compressive strain values.

Both sensor types give average values of strain along their gauge lengths. Finite element method (FEM) results confirmed that in the upper and lower surfaces the strain was constant in the region between the inner rollers in the linear elastic regime, and even for the maximum experimental plastic deformation, the difference between the strain results for the centre and the extremity of a sensing length of $10 \mathrm{~mm}$ was $0.47 \%$. Therefore the influence of the different sensor lengths $(3.18 \mathrm{~mm}$ in the case of electrical strain gauges and $5 \mathrm{~mm}$ in the case of the FBG sensors) is negligible and this calibration procedure is applicable.

The specimen is a $180 \mathrm{~mm}$ long beam of rectangular cross section $45 \mathrm{~mm}$ wide and $3 \mathrm{~mm}$ high. The specimen is loaded by rollers of $30 \mathrm{~mm}$ diameter on the upper surface and $20 \mathrm{~mm}$ diameter on the lower surface. For the calibration of $5 \mathrm{~mm}$ long FBG sensors, Vishay CEA-13-125UN-120 strain gauges with a gauge length of $3.18 \mathrm{~mm}$ were used. The specimen bending was carried out on a servo-hydraulic MTS machine equipped with a $250 \mathrm{kN}$ load cell at constant room temperature under displacement control. The actuator speed was $1 \mathrm{~mm} \mathrm{~min}^{-1}$. After plasticity occurred, the movement of the actuator was inverted, and the test ended when the specimen was no longer in contact with the actuator.

\subsection{Calibration for temperature measurements}

The FBG sensors were calibrated using type-K thermocouples in a temperature-controlled oven. It is relatively easy to isolate the thermal effects from strain effects by the application technique described in section 1.1.

Temperature measurement calibration for the FBG sensors was performed in an oven capable of reaching temperatures between -70 and $200{ }^{\circ} \mathrm{C}$, which on the one hand is enough for the intended range to be measured during the welding experiments, and on the other hand is very near the maximum temperature which the adhesive used for sensor application can withstand. Three sensors were applied on a $360 \times 250 \mathrm{~mm}^{2}$ and $3 \mathrm{~mm}$ thick plate, using the same aluminium and bonding technique as in other experiments.

\subsection{Results}

Figures 2 show the calibration curves obtained for the strain and temperature sensors. Values for the temperature calibration curve were recorded in both the heating and cooling phases of the experiment. In the case of the 


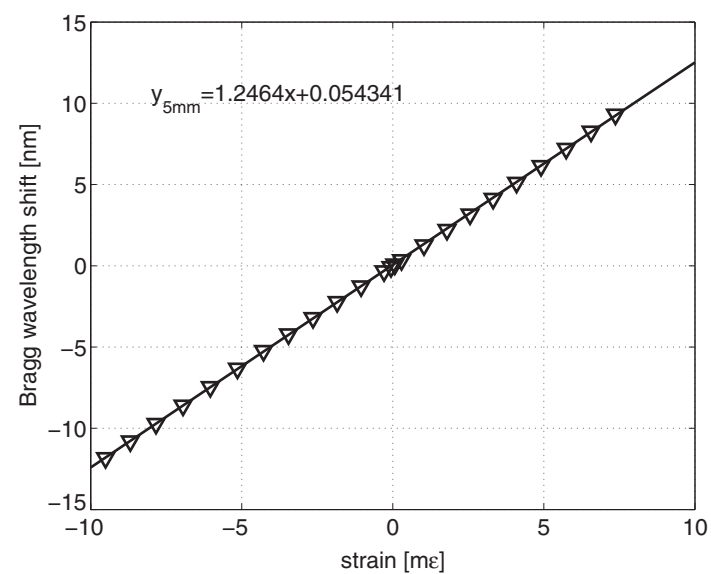

(a)

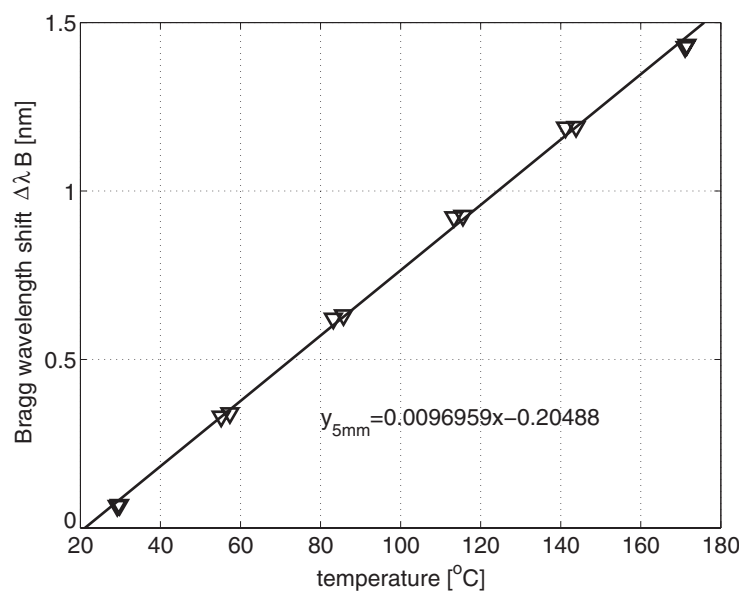

(b)

Figure 2. FBG sensor calibration curves for FBG $5 \mathrm{~mm}$ long gratings bonded using Vishay M-Bond 600 strain gauge adhesive. (a) Strain. (b) Temperature.

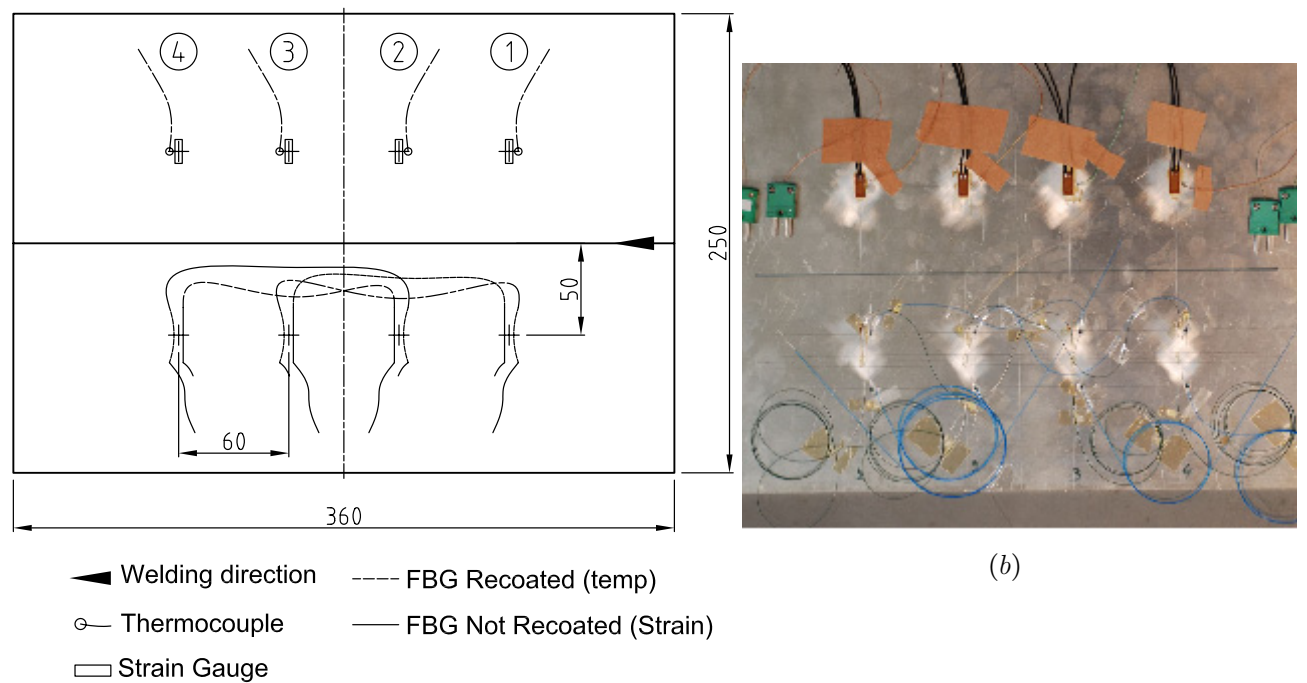

$(a)$

Figure 3. Instrumentation scheme for the MIG welding experiment. (a) Scheme. (b) Picture.

strain calibration curve, data were recorded on the upper surface for tensile and lower surface for compressive strain simultaneously.

For small displacements, the behaviour of the specimen is linear elastic, but progressively diverges from the linear elastic model. It should be noted that both sensor types (electrical strain gauges and FBG sensors) measured the full range of applied strain during the experiments. A nearly linear relationship between the Bragg wavelength shift and temperature was found.

\section{Welding monitoring}

As shown in the previous section, the $5 \mathrm{~mm}$ long FBG sensors lead to valid calibration data and were therefore used for welding monitoring.

MIG was chosen as the first technique to be monitored since it is a common process, capable of welding the studied aluminium alloy in a robotic configuration, and it permits testing the sensors in harsh environments. While the FBG sensors have been shown to work when installed on the lower surface of the plate by Suárez et al [5], in the present case the less protected upper surface, where the welding radiation is more apparent, was instrumented.

A second set of experiments was then performed on FSW due to the high current interest in this solid-state welding process. The instrumentation space is reduced due to its strong clamping requirements. This presents a difficult challenge to other monitoring techniques, such as digital image correlation-based techniques, but may be overcome using FBG sensors.

\subsection{Instrumentation}

3.1.1. MIG experimental setup. MIG welding was performed on a $360 \times 250 \mathrm{~mm}^{2}$ wide and $3 \mathrm{~mm}$ thick AA6082T6 plate. Figure 3 shows the instrumentation scheme used in this experiment and a picture of one of the specimens. 


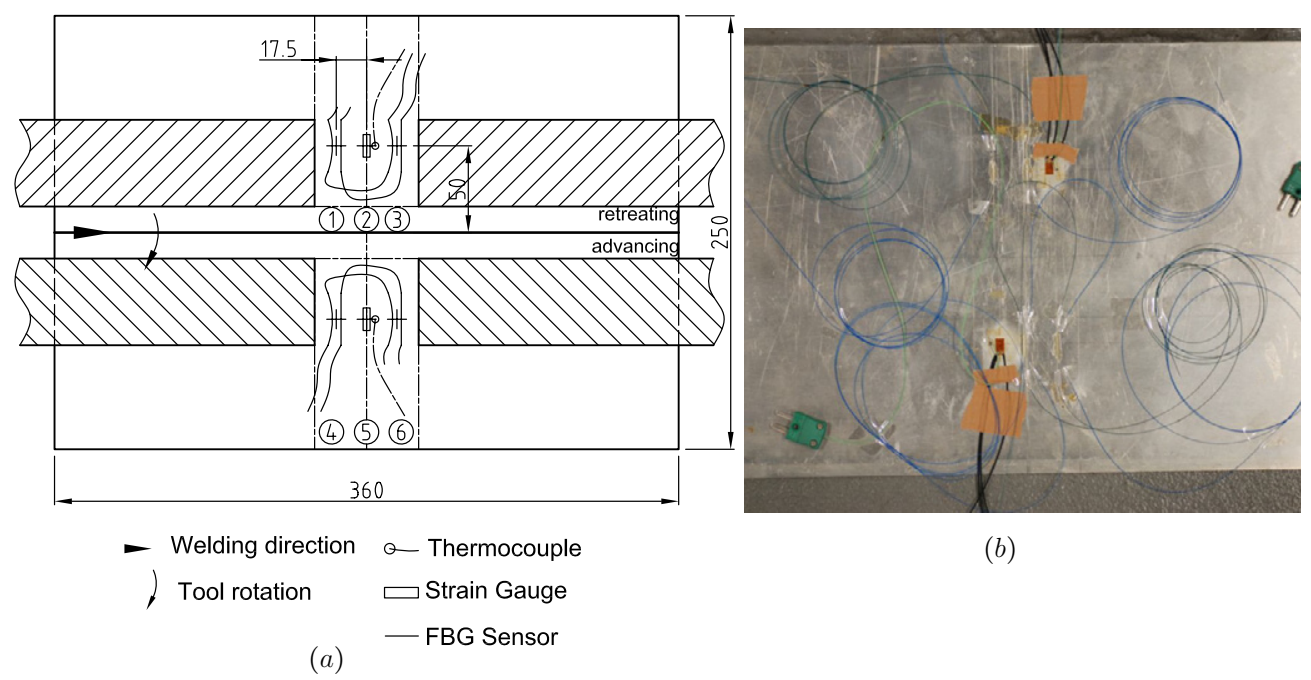

Figure 4. Instrumentation scheme for the FSW welding experiment (shaded: gripping area). (a) Scheme. (b) Picture.

Table 1. MIG welding parameters

\begin{tabular}{ll}
\hline Parameter & Value \\
\hline Welding speed & $700 \mathrm{~mm} \mathrm{~min}^{-1}$ \\
Stick-out & $20 \mathrm{~mm}$ \\
Current & $128 \mathrm{~A}$ \\
Arc voltage drop & $17.1 \mathrm{~V}$ \\
\hline
\end{tabular}

Four strain gauges and four thermocouples were applied, and their results were compared with data of four temperaturemeasuring fibres and four strain-measuring fibres. All fibres were protected with a silicon-based compound as used by Moreira et al [6], and strain gauges were protected with a dedicated strain gauge protective coating, Vishay 3145 RTV. The thermocouples and temperature-measuring FBG sensors were covered with silver-based thermal compound for better heat transfer before the silicon-based protective cover was applied for radiation protection.

Vishay M-Bond 600 was used for bonding the strainmeasuring fibres and the strain gauges. The specimen was subjected to a post-cure for the adhesive for $2 \mathrm{~h}$ at $200^{\circ} \mathrm{C}$. This additional procedure introduces some problems for real world applications, since the instrumentation process takes longer, and the exposure to such temperatures does significantly affect the heat treatment T6 of the alloy AA6082, as shown by Marioara et al [12].

3.1.2. FSW experimental setup. FSW was performed on a $360 \times 250 \mathrm{~mm}^{2}$ and $3 \mathrm{~mm}$ thick AA6082-T6 plate. Figure 4 shows the instrumentation scheme used in this experiment and a picture of one of the specimens.

The same bonding technique and material was used for FSW as was used for MIG welding. Due to the special bonding technique used and the very small diameter of the optical fibres, temperature and strain may be measured almost at the same location.

3.1.3. Welding parameters. MIG welding was performed with the parameters presented in table 1 .
Table 2. FSW parameters

\begin{tabular}{ll}
\hline Parameter & Value \\
\hline Welding speed & $290 \mathrm{~mm} \mathrm{~min}^{-1}$ \\
Rotation speed & $1500 \mathrm{rpm}$ \\
Plate thickness & $3.02 \mathrm{~mm}$ \\
Penetration & $2.97 \mathrm{~mm}$ \\
Shoulder diameter & $15 \mathrm{~mm}$ \\
Pin & Conical threaded pin, $\phi=5 \mathrm{~mm}$ \\
\hline
\end{tabular}

FSW was performed under displacement control with the parameters presented in table 2 .

\section{2. $M I G$}

Two specimens were welded and monitored by the MIG welding process. Data were recorded during the complete weld until clamping release.

3.2.1. Results. The harshness of the MIG process poses interesting challenges, which have been mainly overcome in this work.

While the temperature profiles measured by electrical and optical sensors were similar, the strain profiles were different, which may be explained by the fact that, in contrast to the FBG sensors, electrical strain gauges are self-temperature compensated. Self-temperature compensation is achieved by selecting a strain gauge grid material which has the same thermal expansion coefficient as the specimen material itself. The strain gauge will therefore expand with the specimen without affecting the resistance of the grid significantly when only thermal loads are applied. This is not possible with the used optical sensors which measure the thermal expansion of the specimen additionally to the mechanically induced strain.

One possibility, to compare the results considering this effect, is to look only at the residual strain value, which is quite similar in the present case after the plate has cooled down. Another approach is shown in figure 5 where the theoretical thermal expansion of the aluminium measured by 


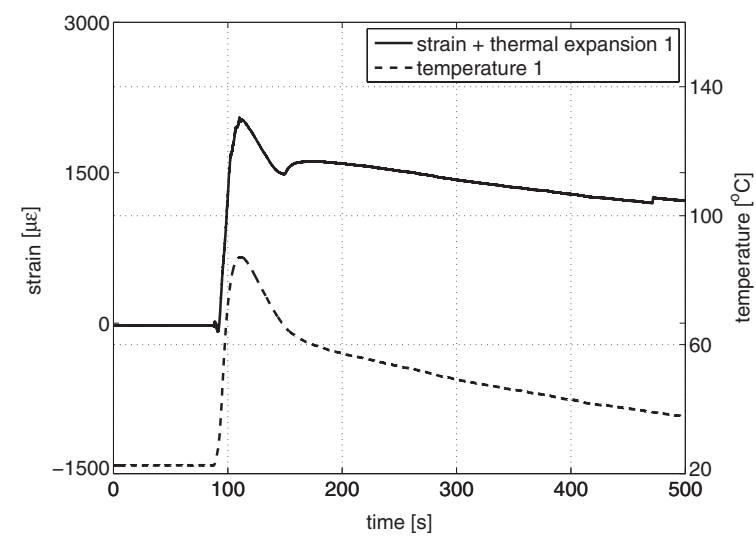

$(a)$

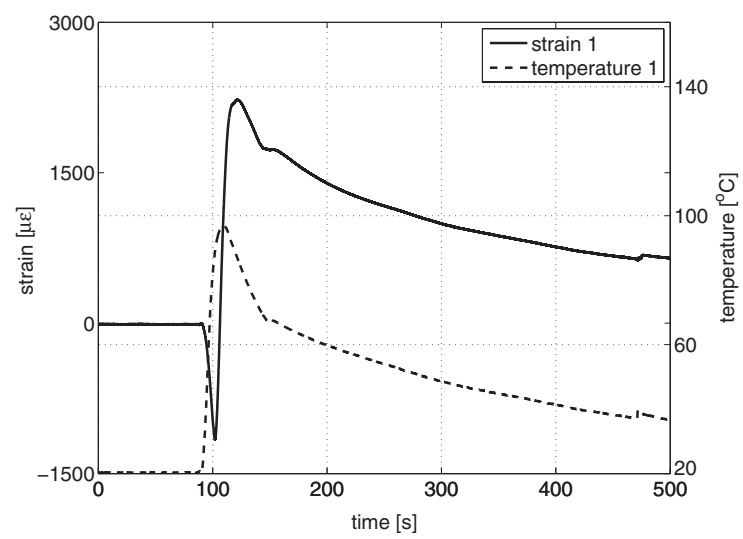

(b)

Figure 5. Comparison of the total strain measured by the electrical and optical sensors during the MIG welding experiment. (a) Electrical sensors: measured strain + theoretical thermal expansion $(b)$ FBG sensors: measured strain.

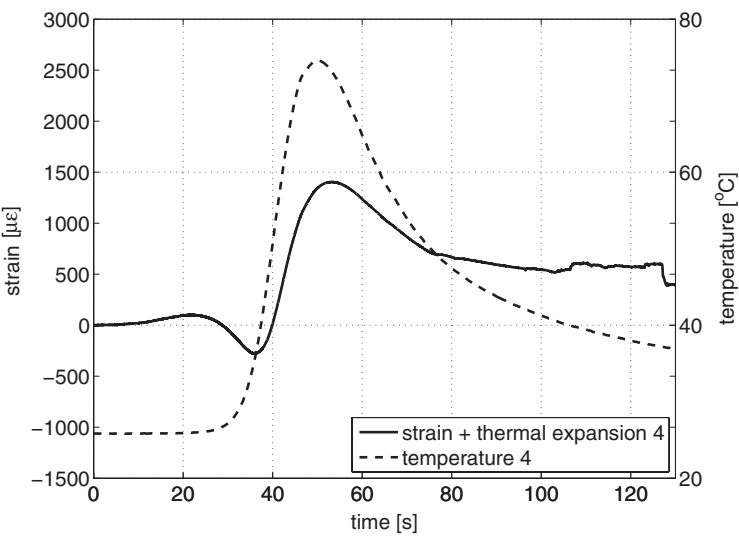

(a)

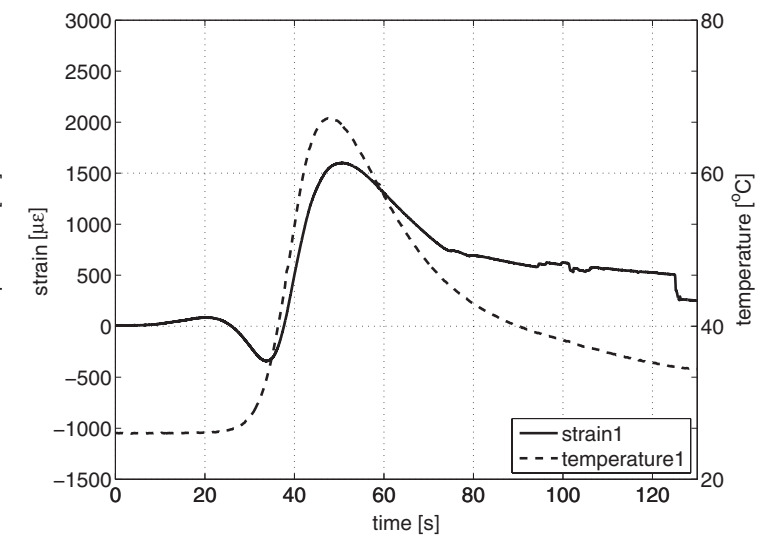

(b)

Figure 6. Comparison of the total strain measured by the electrical and optical sensors during the FSW experiment. (a) Electrical sensors: measured strain + theoretical thermal expansion. (b) FBG sensors: measured strain.

the thermocouple is added to the mechanical strain measured by the self-temperature compensated strain gauge.

This approach leads to similar results between both measuring techniques for the transient and residual strains and also for the temperature measurements. The thermal expansion has an offset in the negative temporal direction when compared to the FBG sensors, since the thermocouples were placed approximately $5 \mathrm{~mm}$ before the centre of the strain gauges in the welding direction. This can explain why in figure $5(a)$ the strain seems to start rising earlier than in figure $5(b)$ in relation to the temperature curves.

\subsection{FSW}

Two specimens were welded using this welding technique. Measurements were started after clamping and stopped only after clamping release, which allows the measurement of the residual strain introduced by the welding process.

3.3.1. Results. Since the difference obtained between strain sensors based on optical fibres and electrical sensors is severe in the region of high thermal loads, the thermal expansion is added to the strain measured by electrical strain gauges for comparability reasons as already justified, see figure 6 .

\subsection{Comparison of strain and temperature in both welding processes}

Both welding processes may be compared in a variety of ways. While the base material is the same in both cases and the final result is a defect-free welded plate, the welding speed of the MIG welding process was significantly higher. Nevertheless, a comparison of the main strains and temperatures between both welding processes based on the FBG sensor measurements can be made. Measurements were made at comparable sites on the plate, at the same distance to the welding line $(50 \mathrm{~mm})$.

When comparing the temperatures in the MIG weld, shown in figure 5, and the FS weld, shown in figure 6, it can be seen that at a distance of $50 \mathrm{~mm}$ to the weld, the measured peak temperatures in the fusion process are approximately $70 \%$ higher than in FSW. Additionally, the time from peak temperature to a temperature below $40{ }^{\circ} \mathrm{C}$ is around $40 \mathrm{~s}$ in FSW, while for the MIG welding process it takes almost ten times as long, which means that significantly more heat was introduced by the MIG welding process. 
In terms of strain, it can be seen from figure 5 for MIG and figure 6 for FSW, that the residual strain, which is the strain left in the plate after cooling down, is between two and three times higher in the MIG than in the FSW process. This justifies the lower distortion found in the FSW plate.

During the welding processes, in the case of MIG welding, the tensile strains measured with strain gauges, which do not include the thermal expansion of the material, are also higher than in the FSW process, while the compressive strains are almost identical. The compression in the case of FSW most likely is originated by the lateral forces created by the tool which penetrates the material and therefore, partially displaces it to the sides compressing the clamped material. After the tool passes, the strains turn into low tensile strains, eventually reaching almost zero after the clamping is released. In the case of the MIG welding process, tensile strains appear in the cooling down phase due to the contraction of the added material in the centre. The compression seen when the torch passes the strain gauges is due to the higher volume of the fused addition and parent material which compresses the restrained plate outside the bath area where the strain gauges are placed.

Looking at the results measured by the FBG sensors, which include both the specimen mechanical strain and the thermal strain, significantly higher total strains are measured during the MIG welding process, which are related to the higher thermal energy introduced by the MIG welding process. This high stress together with the high gradient expected in this fusion welding process is most likely mainly responsible for the distortion observed in the plates, and should therefore be monitored in a variety of conditions, using simple and costeffective instrumentation systems such as FBG sensors. The multiplexing capabilities of the FBG sensors make it possible to acquire even more data points with a single fibre.

\section{Concluding remarks}

In this work the use of FBG sensors was demonstrated for monitoring the MIG and FSW processes obtaining good results.

Before the welding experiment, the sensors were calibrated. The use of the four-point bending test to calibrate the FBG sensors along a large range of positive and negative strain values was presented. Since FBG sensors and electrical strain gauges measure an average strain along the gauge length, it is important to know if strain in that region is constant or not. Therefore a finite element model for simulating the plastic bending deformation of a beam subjected to a four-point bending test was successfully created using Abaqus. The analysis of the surface strain distribution along a $10 \mathrm{~mm}$ long region at mid span was carried out showing that strain is essentially constant in that region, for each loading step considered, validating this calibration approach. Measurements by strain gauges have been successfully used for validating numerical data. The calibration curve for the FBG sensors with a gauge length of $5 \mathrm{~mm}$ measuring strain between -10 and $10 \mathrm{~m} \varepsilon$ was determined.
FBG sensors for measuring temperature were successfully calibrated using an oven, taking into account the material and adhesive later used in the welding experiment. The calibration curve for the FBG sensors measuring temperature between 30 and $180{ }^{\circ} \mathrm{C}$ has been obtained successfully.

It has been shown that $5 \mathrm{~mm}$ long gratings lead to valid temperature and strain measurement results. Since shorter gratings are recommended for high gradient fields, welding monitoring was performed with these gratings.

During MIG and FSW, strain and temperature were successfully monitored by strain gauges, thermocouples and FBG sensors, demonstrating the ability of the FBG sensor installation to withstand harsh environmental conditions while leading to trustworthy results. During FSW it was shown that this kind of instrumentation complies with the strong clamping requirements of this joining process.

The sensors were able to withstand harsh conditions while acquiring signals, paving the way for FBG-based SHM. These sensors may be installed on the base material and monitor component lives from the beginning, through the production process until the end of life of a structure. A good knowledge of the actual stress state inside a structure would therefore be obtained, optimizing condition-based maintenance procedures.

The next steps when using FBG-based sensors for measuring temperature and strain during welding should be distributed strain sensing, since this could potentially reduce or even eliminate problems related to the high gradients found in welding situations and at the same time enrich the information gathered.

Possible protection of the fibres during welding applications have to be studied for future usage, since the unprotected fibres are very fragile. Although it was shown in this work that these sensors are capable of measuring strain and temperature during different welding processes, for industrial use the protection of the bare fibres has to be stronger in order to avoid the destruction of the sensor during handling. Such a protection scheme could afterwards be extended to be left in place for SHM applications. Future work will include packaging to withstand industrial conditions.

\section{Conclusions}

A calibration suitable for a wide range of compressive and tensile strains was proposed in the present paper.

An improved technique for FBG temperature sensor placement was used, which allows the strain and temperature effects to be isolated while simultaneously retaining the ability to use multiple sensors on a single fibre.

Application of FBG sensors for welding monitoring, previously described for arc welding processes, was now demonstrated for FSW. Both for strain and temperature, fibres with several inscribed gratings were used for data acquisition along the weld, perpendicular to the welding direction. $5 \mathrm{~mm}$ long gratings were used for measurement of strain and temperature in the weld bead side of the MIG welded and shoulder side of the FSW plates. 


\section{Acknowledgments}

The present work was partially funded by the project PTDC/EME-TME/66362/2006 and PhD scholarship SFRH/BD/41061/2007 of the Portuguese Fundação para a Ciência e Tecnologia. Dr P Moreira acknowledges POPHQREN-Tipologia 4.2 promotion of scientific employment funded by the ESF and MCTES. The help of M A V de Figueiredo and J F R Almeida is gratefully acknowledged.

\section{References}

[1] Tennyson R 2001 Installation, use and repair of fibre optic sensors ISIS Canada Design Manual No. 1

[2] Barbosa C, Costa N, Ferreira L, Araújo F, Varum H, Costa A, Fernandes C and Rodrigues H 2008 Weldable fibre Bragg grating sensors for steel bridge monitoring Meas. Sci. Technol. 19125305

[3] Figueiras J, Felix C and Costa B A 2005 Testing and monitoring of a centenary arch bridge Struct. Infrastruct. Eng. 1 63-73

[4] Thursby G, Culshaw B and Betz D C 2008 Multifunctional fibre optic sensors monitoring strain and ultrasound Fatigue Fracture Eng. Mater. Struct. 31 660-73

[5] Suárez J, Remartínez B, Menéndez J, Güemes A and Molleda F 2003 Optical fibre sensors for monitoring of welding residual stresses J. Mater. Process. Technol. 143-144 316-20

[6] Moreira P M G P, Frazao O, Tavares S M O, de Figueiredo M A V, Restivo M T, Santos J L and de Castro P M S T 2007 Temperature field acquisition during gas metal arc welding using thermocouples, thermography and fibre Bragg grating sensors Meas. Sci. Technol. 18 877-83

[7] Ferraro P and de Natale G 2002 On the possible use of optical fiber Bragg gratings as strain sensors for geodynamical monitoring Opt. Lasers Eng. 37 115-30

[8] Duck G, Renaud G and Measures R 1999 The mechanical load transfer into a distributed optical fiber sensor due to a linear strain gradient: embedded and surface bonded cases Smart Mater. Struct. 8 175-81

[9] Richter-Trummer V 2008 Characterization of different aluminium alloys of the series 6000 and of their joining processes MIEM Thesis Faculdade de Engenharia da Universidade do Porto

[10] Richter-Trummer V, Silva S O, Frazão O, Tavares S M O, Moreira P M G P and de Castro P M S T 2009 O ensaio de flexão em 4 pontos para calibração de deformação usando sensores de Bragg OERN 5-5 Encontro Nacional do Colégio de Engenharia Mecânica da Ordem dos Engenheiros (Guimarães, 13-14 February) (in Portuguese)

[11] Timoshenko S and Goodier J 1951 Theory of Elasticity 2nd edn (New York: McGraw-Hill)

[12] Marioara C, Andersen S, Jansen J and Zandbergen H 2003 The influence of temperature and storage time at RT on nucleation of the $\beta^{\prime \prime}$ phase in a $6082 \mathrm{Al} \mathrm{Mg-Si}$ alloy Acta Mater. 51 789-96 